\title{
Populäre Kultur, populäre Religion und die Refiguration der Gesellschaft
}

\author{
Hubert Knoblauch
}

Eingegangen: 15. Mai 2021 / Überarbeitet: 19. August 2021 / Angenommen: 21. August 2021 / Online publiziert: 15 . Oktober 2021

(C) Der/die Autor(en) 2021

Zusammenfassung In diesem Beitrag soll der Begriff der populären Religion in einem soziologischen Zusammenhang erläutert und ins Verhältnis zu dem setzen, was als populäre Kultur bezeichnet wird. Dabei sollen nicht nur die Unzulänglichkeiten des Konzeptes der populären Kultur angeschnitten werden. Auch der Begriff der populären Religion bedarf einer weiteren Ausarbeitung. Dabei ist neben der zunehmenden Marktorientierung der Religion und ihrer Verbindung mit den Massenmedien vor allem die digitale Mediatisierung hervorzuheben. die nicht nur die Religion verändert. Die damit einhergehenden Veränderungen werden als „Refiguration“ skizziert. Diese wurde bisher mit Blick auf gesamtgesellschaftliche Phänomene wie auch auf räumliche Veränderungen untersucht und soll hier - in einem ersten, kurzen und vorläufigen Entwurf - nun auf die religiöse Entwicklung übertragen werden.

Schlüsselwörter Refiguration · Populäre Kultur · Populäre Religion ·

Mediatisierung $\cdot$ Kommunikativer Konstruktivismus $\cdot$ Religionssoziologie

Hubert Knoblauch $(\bowtie)$

Technische Universität Berlin, Fachgebiet Allgemeine Soziologie, Sekretariat FH 9-1,

Fraunhoferstraße 33-36, 10587 Berlin, Deutschland

E-Mail: hubert.knoblauch@tu-berlin.de 


\section{Popular culture, popular religion and the refiguration of society}

Abstract In this paper the concept of popular religion will be explained in a sociological context and put in relation to what is called popular culture. In doing so, not only the shortcomings of the concept of popular culture will be touched upon. The concept of popular religion also needs further elaboration. In addition to the increasing market orientation of religion and its connection with the mass media, digital mediatization should be emphasized which has massive effects also on religion. The accompanying changes are outlined as "refiguration". Refiguration has so far been examined with a view to phenomena in society as a whole as well as to spatial changes and will now be applied here-in a first, brief and preliminary draft - to the recent changes in religion.

Keywords Refiguration · Popular Culture · Popular Religion · Mediatization · Communicative Constructivism · Sociology of Religion

\section{Einleitung}

Dieses Special Issue der Zeitschrift für Religion, Gesellschaft und Politik wie auch die ihm vorausgegangene Jahrestagung der Sektion Religionssoziologie der Deutschen Gesellschaft für Soziologie sind dem Thema „Religion und Populärkultur“ gewidmet. Die Themenstellung spielt indirekt auf den Begriff der ,ppopulären Religion“ an, der auch im Call for Papers zur Tagung wie auch im Editorial ausdrücklich erwähnt wird. Weil der Begriff in einigen der Beiträge in einem Sinne verwendet wird, wie er in einigen meiner Arbeiten entwickelt wurde, möchte ich diesen Begriff hier in einem soziologischen Zusammenhang erläutern und ins Verhältnis zu dem setzen, was als populäre Kultur bezeichnet wird. Dabei möchte ich deutlich machen, wie unbefriedigend und begrenzt der Begriff der populären Kultur ist. Allerdings bedarf auch der Begriff der populären Religion einer weiteren Ausarbeitung, die die empirisch beobachtbaren Veränderungen der Religion, aber auch der Gesellschaft als Ganze in den Blick nimmt. Wenn wir die wesentlichen Züge der Religion betrachten, hat sich, neben der zunehmenden Marktorientierung der Religion und ihrer Verbindung mit den Massenmedien, vor allem die digitale Mediatisierung als ein Prozess erwiesen, der (nicht nur) die Religion verändert. Dies wurde an anderer Stelle als „Refiguration“ skizziert. Die digitale Mediatisierung ist sicherlich nur eine Kraft unter vielen, doch ist sie diejenige, die auch im historischen Vergleich zweifellos neue Züge aufweist, so dass ich sie besonders hervorheben werde.

Weil ich das Konzept der populären Religion in einem umfänglichen Buch ausgearbeitet und darin wie auch in späteren Arbeiten empirisch spezifiziert habe, möchte ich diese Erläuterungen des Begriffs der populären Religion hier nicht in Form eines ausführlicheren Aufsatzes entfalten und empirisch belegen. ${ }^{1}$ Seit der Formulierung der These der populären Religion vor knapp 25 Jahren habe ich eine Reihe von

\footnotetext{
${ }^{1}$ Zur Zeit der Abfassung dieses Textes ist meine Universität Opfer einer Cyberattacke, was dazu führt,
} dass zeitweilig weder die privaten Rechner noch die darin enthaltenen Daten verwendet werden dürfen. 
konzeptionellen Ausarbeitungen, empirischen Untersuchungen und Weiterführungen (etwa zu populären Spiritualität) veröffentlicht, auf die ich hier im deswegen vergleichsweise ausführlichen Anmerkungsapparat verweisen möchte. Bei dem hier sehr knapp und sehr dicht geschriebenen Versuch einer Klärung des Begriffs der populären Religion und seines Verhältnisses zur populären Kultur soll es allerdings nicht nur um einen Rückblick gehen, sondern eher um eine Art Zwischenbilanz. Die Entwicklung der Formen populärer Religion stellt, wie argumentiert werden soll, einen laufenden Prozess dar, so dass der Begriff selbst wie das damit verbundene Bündel an Phänomenen weiter Gegenstand der empirischen Forschung bleiben wird. Aufgrund der Unschärfe des Begriffs des Populären und seiner mangelnden Unterscheidungsmöglichkeit vom „Popularen“ sowie aufgrund neuer Formen der digitalen Mediatisierung erscheint es dabei sinnvoller, diesen beständigen Wandel als Refiguration zu fassen.

\section{Zur Entwicklung des Begriffs}

Der Begriff der populären Religion schließt in einer sehr direkten Weise an Überlegungen an, die Thomas Luckmann $(1967,1991)$ unter dem Titel der „unsichtbaren Religion“ vorgestellt hat. „Unsichtbar“ erschien diese Religion nämlich nicht, weil sie aus dem öffentlichen Raum verschwinde, wie etwa Casanova (1994) gegen diese Religion vorbrachte. Vielmehr betonte Luckmann (1988) sehr deutlich, dass die Religion eine „massenkulturelle Form“ annehme, die jedoch nicht mehr hinsichtlich ihrer „sozialen Formen“ (Praktiken, Rituale, Organisations- und Wissensformen) notwendig als „Religion“ erkennbar sei. Diesen paradoxen Zusammenhang einer „Religion, die sozial nicht als Religion erkennbar ist“", habe ich zunächst (Knoblauch 1997, 2000) auf den Begriff der „Sichtbarkeit der unsichtbaren Religion“ gebracht. Empirische Belege fand ich dafür u.a. in den neuen Formen des New Age, der neuen „Kulte“ und dessen, was mir im Zusammenhang mit den Nahtoderfahrungen als neue Spiritualität erschienen war (Knoblauch 1989, 1999, 2010), greifbar. Der Begriff der Spiritualität sollte nach den Veröffentlichungen von Gebhardt et al. (2005) sowie Heelas und Woodhead (2005) auch in der deutschsprachigen religionswissenschaftlichen Debatte eine immer größere Rolle spielen und so rasch popularisiert werden, dass die Beobachtung einer ,populären Spiritualität“ sich geradezu aufdrängte (Knoblauch 2009). Die populäre Spiritualität entsprach durch ihren sozusagen alternativen Zug auch den Mustern der Luckmannschen unsichtbaren Religion, die sich gerade im deutschsprachigen Raum auf eine höchst sichtbare Weise entfaltet hatte (Klinkhammer und Tolksdorf 2015). In der deutschsprachigen Debatte rückte die Frage nach der Popularität der Religion etwas später als im englischsprachigen Raum in den Blick, weil hier die neueren religiösen sozialen Entwicklungen lange vor dem Hintergrund eurozentrischer Modernisierungstheorien betrachtet wurden und dort die Rolle der populären Kultur durch die „Cultural Studies“ intensiv behandelt wurde.

Für den Wechsel der Perspektiven auf die Religion als etwas, das nicht einfach von Modernisierungsprozessen verdrängt werde, spielten auch in Europa die Anschläge am 11.09.2001 eine zumindest symbolisch entscheidende Rolle: Sie machten der bis 
dahin zumindest zu großen Teilen stark säkularisierungstheoretisch orientierten Religionssoziologie, dem sozialwissenschaftlichen Diskurs zum Thema wie auch der journalistischen und kritischen Öffentlichkeit die Bedeutung der Religion für die Moderne deutlich. Das führte zu einem religionssoziologischen Perspektivenwechsel einiger namhafter Autoren (z. B. Habermas 2001), die mit ihrem publizistischen Gewicht den bis dahin erarbeiteten religionssoziologischen Forschungsstand etwa zur Säkularisierung geflissentlich und nachhaltig übertönten. Es ist durchaus ein Ausdruck der Popularisierung des wissenschaftlichen Diskurses, dass sogar so prominente und elaborierte Kritiken der Säkularisierungstheorie, wie die von Thomas Luckmann, so leichthin übergangen und die „Postsäkularität“ als Konzept des öffentlichen Diskurses auch in der Wissenschaft als ein völlig neuer Zugang dargestellt werden konnte. Der Wechsel der Perspektive zeigt sich auch an regelrechten religionssoziologischen Konversionen, wie etwa der von Peter Berger (1999), der von der Position eines ,methodologischen Atheismus“ kommend nun den Säkularismus der Wissenschaft aufs Korn nahm. Für die Forschung aber rückten nun vor allen Dingen eben jene Züge der organisierten Religion (und von sich als religiös verstehenden Organisationen) in den Blick, die nicht von der Moderne verdrängt wurden, sondern ihr vielmehr ihren Erfolg verdankten. Dazu zählte, was damals noch „Fundamentalismus“ genannt wurde, also bestimmte Strömungen innerhalb des Islam, Hinduismus, Buddhismus und (evangelikalen, neopflingstlerischen, charismatischen) Christentums, deren zunehmende Ausbreitung (damals: außerhalb Europas) quantitativ belegt wurde (Knoblauch 2002).

Die „Popularität“ der Religion zeigte sich also schon in ihrer schieren Verbreitung und wachsenden Ausbreitung, die sich auch auf als hoch-, spät- und postmodern geltende Gesellschaften und Regionen erstreckte, wie etwa die USA und - sofern die populäre Spiritualität dazu gezählt werden kann - die westeuropäischen Gesellschaften. Allerdings handelt es sich beim Aspekt der schieren („massenhaften“) Verbreitung um einen sehr dünnen Begriff des Populären. Daher muss gefragt werden, wie dieser Begriff zusätzlich qualifiziert werden kann: Ist Religion einfach nur wieder ,ppopulär“, zeichnet sich die Popularität durch weitere, die verschiedenen Regionen und Religionen überschreitende gemeinsame Attribute aus, und handelt es sich dabei um eine neue Form der Religion, also um etwas, das mit dem Konzept der populären Religion gesellschaftsdiagnostisch gefasst werden kann?

\section{Populäre Kultur}

An dieser Stelle wende ich mich dem Konzept des Populären bzw. auch - wie der Titel des Special Issue vorschlägt - der populären Kultur zu, wie er in den Sozialund Kulturwissenschaften verwendet wird. ${ }^{2}$

\footnotetext{
2 Mit dem Verweis auf die Sozialwissenschaften unterstreiche ich zum einen, dass wir uns in einem theoretischen Diskussionszusammenhang bewegen, der sich nicht auf Sinn- und Wissensphänomene beschränkt, sondern auch die damit verbundenen verkörperten Handlungen einbezieht, die als relational auf andere bezogen verstanden werden und die Voraussetzung für Institutionen und soziale Strukturen bilden (Knoblauch 2017). Zum anderen wird damit auch betont, dass die im Weiteren genannten Aspekte des Begriffs
} 
Der Begriff des „Populären“ bildet zwar keinen Kern sozialwissenschaftlicher Forschung. Er ist aber im Rahmen der interdisziplinär angelegten kritischen Theorie und, in ihrer Nachfolge, der „Cultural Studies“ in Weisen elaboriert worden, die auch in die soziologischen, religionssoziologischen und religionswissenschaftlichen Debatten eingingen. Allerdings findet sich innerhalb der Religionssoziologie keine systematische Aufarbeitung dieser Verwendungen. So schlägt Possamai im Englischen zwar 2005 ,,popular religion“ als Konzept vor; er bezeichnet damit aber weitgehend eine massenkulturelle „,consumer religion“. Damit spricht er zwar den für ein Verständnis der Populärkultur unabdingbaren „Marktcharakter“ einer Religion an, die sich zunehmend an der Nachfrage orientiert, Laien damit in Konsumenten und religiöse Organisationen in Anbieterinnen verwandelt (Usinier und Stolz 2014); doch greift er damit eine keineswegs so neue Entwicklung auf, die schon Berger mit seinem berühmten „Marktmodell“ (Berger 1965), Luckmann (1991) und dann auch die Rational Choice- und Tauschtheorien (Bruce 2002) sehr ausführlich untersucht haben. International beachtenswert ist auch das im frankophonen Raum verbreitete Konzept von Isambert (1982), der unter ,,religion populaire“ indessen eher das versteht, was im Deutschen als „Volksreligion“ bezeichnet worden war. Die Umrisse wie auch die Probleme dieses Begriffs in verschiedenen westlichen Sprachkulturen hatten Ebertz und Schultheis (1986) sehr detailliert auseinandergesetzt, die an seiner Stelle das Konzept der ,,popularen Religion“ vorschlugen. Im Unterschied zur eher an traditionalistischen Kulturen orientierten „Volksreligion“ bzw. dem, was im Englischen auch als „Folk-Religion“ bezeichnet und untersucht wird, nimmt dieser Begriff deutliche soziologische Züge an. Während der Begriff der „Folk“-Kultur mit einer ,,irreführenden Homogenität“ (Burke 1988) verbunden ist, bleibt die ,,populare Religion“ in verschiedener Weise von sozialen Ungleichheiten geprägt. In Anlehnung an den ,,populären Geschmack“ der Unterschicht bei Bourdieu (1985) ist die populäre Religion mit sozialen Gruppen verbunden, die über vergleichsweise niedriges symbolisches und ökonomisches Kapital verfügen. In gewisser Weise ähnelt diese Vorstellung der Unterscheidung von „,low brow“ und ,high brow“, wie sie die kritische Theorie entwickelt hat (Kausch 1988), doch bezieht sie sich - insbesondere in Gestalt Adornos - weitgehend auf die populäre Massen-Kultur. Sie ist, soweit ich sehe, ebenso wenig auf die (populäre) Religion übertragen worden wie das etwas anders vorgehende Konzept der populären Kultur in den „Cultural Studies“. Denn die „Cultural Studies“ gehen von einer umfassenden, von den herrschenden Klassen getragenen hegemonialen Kultur aus, die von den anderen Klassen zwar angenommen bzw. ,,angeeignet“" wird. Doch deren anders gelagerte soziale Positionen (Interessen, Wissens- und Praxisformen) erzeugen bei der Aneignung der populären Kultur eine Differenz zur hegemonialen Kultur, die sich in einer gewissen Widerständigkeit, Subversivität und Kreativität äußern kann (Fiske 1989). Unter ,,populärer Kultur“" werden in diesem engeren Sinne nur diejenigen musikalischen, filmischen, literarischen und anderen Ausdrucksformen und Objektivationen gefasst, die nach dem Zweiten Weltkrieg zunächst getragen von einer jugendkulturellen Bewegung vor allem vom angelsächsischen Raum ausgingen und sich sowohl

in ihren Qualitäten und, gegebenenfalls, auch in ihrer quantitativen Verbreitung mithilfe von empirischen Daten und nachvollziehbaren Analysemethoden begründet, gestützt, differenziert oder widerlegt werden. 
über den wachsenden Markt als auch die Massenmedien zunächst in der westlichen Welt, zunehmend aber auch im Ostblock ausgebreitet haben. Es handelt sich dabei vor allem um industrielle Massengüter (Poster, Platten) oder lokale Veranstaltungen bzw. kommerzialisierbare und live mediatisierte Events, die sehr stark an die neuen elektronischen Massenmedien gebunden sind, also Film, Fernsehen, Rundfunk, auch wenn sie mit ihren besonderen (etwa in der Musik stark rhythmisierten) Formen und (auch in der Übernahme von im Westen nicht hegemonialen religiösen) Inhalten eine „gegenkulturelle“ Haltung einnahmen.

\section{Populäre Religion}

An dieser Stelle sind wir bei der Frage angekommen, die im Titel formuliert wurde: Wie verhält sich die Religion zur populären Kultur? Diese Frage ist auch entscheidend für ein Verständnis der populären Religion. Denn es gibt zwar eine Reihe von Versuchen, die „Pop-Religion“ als etwas zu fassen, das in der populären Kultur auftritt (Greeley 1993) - etwa als Übernahme religiöser Symbole, Gesten und musikalischer Elemente in der populären Musik oder populärer Elemente in der Religion (wie z. B. die Nutzung der Popmusik in evangelikalen Gottesdiensten oder von Marketingtechniken für religiöse Organisationen und Veranstaltungen), doch handelt es sich hier nur sozusagen um Oberflächenphänomene dessen, was ich als populäre Religion bezeichne. Religion weist dabei alle Züge der populären Kultur auf. Im religiösen Bereich ist die industrielle Massenproduktion schon seit dem frühen 19. Jahrhundert, ja in gewisser Weise schon seit dem Buchdruck verbreitet, so dass der eigentlich neue Aspekt der modernen Populärkultur mit dem Einsatz von elektronischen Massenmedien in und für die Religion verbunden ist (Karstein 2019). Auf diese Verbindung verweist auch die Bezeichnung ,electronic church“, und eine ihrer exemplarischen Ausdrucksformen bilden die Veranstaltungen von Billy Graham (vgl. Knoblauch 2019). Seit den 1940er-Jahren setzt er gezielt das damals neue Massenmedium des Fernsehens ein, um seine Predigten live an ein Millionenpublikum zu übertragen bzw. es durch die mediale Rezeption zu missionieren. Gleichzeitig verwandelt Graham die Gottesdienste in Massenveranstaltungen mit zehn-, ja hunderttausenden an Teilnehmenden, die sich vor Ort im Stadion befinden. Sicherlich sind religiöse Massenveranstaltung keineswegs ein Novum in der Religionsgeschichte. Grahams Veranstaltungen zeichnen sich aber nicht nur dadurch aus, dass er seine Gottesdienste in den „Tempeln“ der modernen populären Kultur abhielt, also Sportstadien (wie dem Madison Square Garden in den 1940er-Jahren oder dem Berliner Olympiastadion 1951). Seine Innovation besteht vielmehr darin, dass diese Veranstaltungen zum einen durch Film und Fernsehen übertragen wurden (wie dies etwa Leni Riefenstahl schon am selben Ort wenige Jahre zuvor machte). Zum zweiten wurden die Fernsehkameras und das dadurch repräsentierte Publikum selbst zum integralen Bestandteil der Veranstaltung. Erst damit handelt es sich um ein Event in einem prägnanten Sinne, weil Grahams eigene Handlungen während der Gottesdienste von den Medien mit geprägt werden und weil das Live-Publikum selbst wieder zum Objekt eines medialen Publikums wird (Haken 2017). 
Diese Hinweise machen die Rolle der Medien deutlich, die nicht einfach als Verbreitungsinstrumente dienen, wie es etwa die für Handlungen so unsensible Systemtheorie vermutet. Events machen vielmehr deutlich, dass die Handlungen selbst durch die Nutzung der Medien verändert werden. Sie nehmen also nicht nur eine neue räumliche Struktur an, da sie ja den Körper sozusagen verlängern und Handlungen über den Raum hinweg bis in die größten Weiten vermitteln können, sondern schaffen auch eine zeitliche Präsenz, die mit dem neudeutschen Begriff „live“ gefasst werden kann (Auslander 1999). Das Konzept der Mediatisierung, das von Hepp und Krotz (2012) entwickelt und von ihnen selbst schon auf religiöse Events angewandt wurde (Forschungskonsortium WJT 2007; Hepp und Krönert 2009), stellt eine zentrale Neuerung dar, um das Phänomen der populären Religion zu verstehen. Mediatisierung sollte dabei keineswegs nur auf die technischen Kommunikationsmedien beschränkt werden, sondern schließt auch die materiale Kultur mit ein und ihre massenindustrielle oder neuerdings auch individualisierte und ,singularisierende“ (Reckwitz 2017) Produktion. Und auch wenn wir die Digitalisierung als eine besondere Neuerung betrachten müssen, so sollten auch die ,,alten“ Massenmedien nicht aus dem Blick geraten, die nach wie vor Teil des „Medienmix“ sind. So gehören das Radio und das Fernsehen immer noch zum Medienrepertoire in den meisten Kulturen und Gesellschaften, auch wenn diese Medien sich selbst etwa zum digitalen Radio oder zum von Internetfirmen produzierten und vermittelten Fernsehen gewandelt haben. Damit begründet die digitale Mediatisierung, dass sich bei der gegenwärtigen populären Religion vermutlich auch historisch völlig neue Aspekte entfalten, die in ihren ersten Konzeptionalisierungen um das Jahr 2000 so noch nicht absehbar waren. ${ }^{3}$

Diese Mediatisierung meint nicht nur, dass der vergleichsweise marginale Anteil der Teilnehmenden an Gottesdiensten im Fernsehen nun durch eine vermutlich nicht beeindruckendere Zahl an „Klicks“ für Gottesdienste in Youtube ergänzt wird oder dass religiöse Kommunikation nun in den Formaten des Internets stattfindet (Neumaier 2016). Mediatisierung geht über die Rekrutierung der Laien für religiöse Veranstaltungen und Organisationen hinaus, die sich nun über die Algorithmen von Computersoftwares leiten lassen, sich eher an den audiovisuellen Darstellungen orientieren und ihre Kontaktaufnahmen mit Emails oder Bookingsystemen produzieren. Die Erforschung der Digitalisierung des Religiösen befindet sich zwar erst in ihren Anfängen (Merle 2019), jedoch gibt es deutliche Hinweise darauf, dass die digitalen Kommunikationsmedien eigene populäre Formen annehmen. Damit wirken sie sich auch auf die Formen des Populären aus, wie sie noch von den herkömmlichen Massenmedien geprägt waren, und verändern diese Formen. Das wurde u.a. in Untersuchungen aufgezeigt, die im Rahmen unserer Arbeitsgruppen durchgeführt wurden, wie etwa zum Event „Papstbesuch“ (Knoblauch 2014). Beim Besuch des damaligen Papstes Benedikt 2011 in Deutschland und in Berlin zeigen sich - neben den noch immer existierenden massenmedialen Formen - gerade bei dem so sehr auf Rituale achtenden katholischen Papst weitgehende Veränderungen

\footnotetext{
3 Dabei sollte beachtet werden, dass der Phase der Digitalisierung eine mittlerweile häufig übersehene „Informatisierung“ voranging und dass nun auch die Digitalisierung selbst verschiedene Innovationszyklen aufweist. Eine genauere Skizze des Prozesses der Mediatisierung findet sich in Knoblauch (2017).
} 
der Rituale der Frömmigkeit, so dass selbst die Gebetshaltung für Formen der populären Verehrung aufgeben wird. Wie erste analytische Tiefenbohrungen zeigen, wirken sich diese Veränderungen auch auf die Vermittlungswege aus, die nun über die Individualmedien (Handys, Twitter, Whatsapp) verlaufen, und auf die Stile der Frömmigkeit, die selbst produziert (,,prosumer“) werden und stark visuellen bzw. audiovisuellen digitalen Formaten und Mustern folgen (Knoblauch und Blanc 2018). Die auf der Individualnutzung bauenden digitalen Medien erklären auch, warum die populäre Religion mit einer hochgradig subjektivierenden Form der Religiosität, also der Spiritualität, verbunden ist (Knoblauch 2009).

Dass diese Hinweise auf die transformierende Rolle der digitalen Mediatisierung von den Kirchen nur zögerlich aufgenommen (Brunn und Keller 2020) und Versuche zur digitalen Regelung des Kirchenbesuchs und neuen digitalen Formaten so recht erst während der Corona-Krise realisiert wurden, hat aber nicht nur mit einer gewissen Traditionalität zu tun. Diese Zögerlichkeit ist auch Ausdruck davon, dass wir mitten im Prozess der digitalen Mediatisierung stecken, so dass wir die neuen Formen erst noch entdecken und erforschen müssen. Sie ist nicht nur mit einer Vermarktung der Religion verbunden, sondern hängt auch mit einer ihre Massenmediatsierung weiterführenden ,massenhaften Selbstkommunikation“, wie Castells (2009) das nennt, zusammen, die wiederum mit der Präferenz für das einhergeht, was in der Öffentlichkeit als ,spirituell“ bezeichnet wird.

Die Betonung der Rolle der digitalen Mediatisierung sollte jedoch keineswegs als Reduktion des gesamten Wandels nur auf diese Form der Mediatisierung verstanden werden. Das besondere Augenmerk auf die digitale Mediatisierung und die mit ihr verbundenen Veränderungen soll dem (durchaus begründeten) Einwand begegnen, „populäre“ Religion habe es schon immer gegeben. Gerade vor dem Hintergrund der historischen popularen Religion und der Rolle der „,massenkulturellen“ populären Kultur im 20. Jahrhundert darf die Frage gestellt werden, ob es nicht eines anderen Begriffes bedürfte, um diese mit der Digitalisierung verbundenen Besonderheiten und Veränderungen herauszustellen. Dies war auch einer der Gründe, warum das Buch „Populäre Religion“ nicht ins Englische übersetzt wurde, entfällt in der englischen Sprache doch selbst die Unterscheidungsmöglichkeit zwischen ,popular“ und ,populär“. Allerdings müsste auch im Deutschen zwischen dem massenkulturell Populären und dem Populären unterschieden werden, das sich im Zuge der digitalen Mediatisierung zeigt. Um diesen Unterschied zu bezeichnen, habe ich in jüngerer Zeit das Konzept der refigurierten Religion eingeführt (Knoblauch 2020).

\section{Refiguration}

Mit der Zuwendung zum Konzept der Refiguration sollen die Folgen der digitalen Mediatisierung auf die Handlungsweisen - insbesondere ihre veränderte Räumlichkeit - und die damit verbundene Neuordnung sozialer Beziehungen, Institutionen, Infrastrukturen und Interdependenzketten bezeichnet werden (Löw und Knoblauch 2021). Die Mediatisierung ist jedoch keine kausale Ursache der Refiguration, wir haben es also nicht mit einem Technikdeterminismus zu tun, genauso wenig wie die Massenmedien determinierend waren. Vielmehr zeigt sich eine gewisse Wahl- 
verwandtschaft (oder strukturelle Homologie) zwischen den zentralistisch und hierarchisch organisierten religiösen Massenorganisationen und den ähnlich (,,modern“) strukturierten Massenmedien bzw. auch massenmedialen Kommunikationsstrukturen. Refiguration schließt deswegen auch andere Veränderungen mit ein, die mit dem Konzept der populären Religion gemeint sind.

Dazu gehört eine Tendenz, die Gerhards und Neidhart (1990) als „Aufstand des Publikums" bezeichnet haben. Aus systemtheoretischer Sicht beobachten sie schon vor dem Einsetzen der letzten Digitalisierungswelle, dass die Trennung zwischen den Kernen sozialer Funktionssysteme (also auch der Religion, die sie nicht gesondert ansprechen) und dem Publikum dieser Funktionssysteme nun nicht mehr von verschiedenen zwischengeschalteten Ebenen vermittelt wird, sondern dass das Publikum (also z. B. die „Öffentlichkeit““ der Politik) direkt mit dem Kern der Systeme gekoppelt wird. Die Folgen dieser Kopplung für die Politik gerade durch die digitalen Medien sind in beiden Richtungen deutlich zu beobachten (etwa an den „Shitstorms“ gegen machtvolle Repräsentantinnen des politischen Systems, aber auch an popularistischen Tweet-Strategien mit Blick auf die Öffentlichkeit).

Schon vor der Digitalisierung hatte Bourdieu (1985) eine ähnliche Tendenz der Entgrenzung der Funktionssysteme bzw. der Felder beobachtet, die auch leitend für das Konzept der populären Religion wurde. Die massive Zunahme an magischen, spirituellen und religiösen Dienstleistungen betrachtete er im Rahmen seiner Theorie sozialer Felder als Anzeichen für eine „Auflösung des religiösen Feldes“, das an seinen Rändern so durchlässig werde, dass eine klare Zuordnung zur Religion verschwimme.

Die transgressiven Tendenzen zur Überschreitung der Grenzen zum religiösen „System“ oder „Feld“ schienen durch die Digitalisierung mit ihrer neuen Raumlogik der Netzwerke verstärkt zu werden, die flache Hierarchien und Partizipation in allen Bereichen der „Netzwerk-Gesellschaft“ (Castells 1996) versprachen. Allerdings zeigte sich bald, dass die digitale Mediatisierung zu neuen Monopolen führte, die besondere Formen von „Kommunikationsmacht“ (Castells 2009) ausüben. Diese Macht beschränkt sich keineswegs nur auf nicht auf bestimmte ,contents“ angelegte Monopole von global auftretenden Soft- und Hardware-Firmen (Apple, Microsoft, Google etc.), sondern wirkte sich durch Algorithmisierung ebenso wie durch politische Einflussnahmen (etwa in Russland oder China) auf die Verarbeitung von Informationen und damit die Vermittlung von Wissen aus.

Diese der flachen Netzwerklogik entgegenlaufende Logik der Zentralisierung, Kontrolle und Hierarchisierung lässt sich auch im Bereich der Religion beobachten, die mit der Logik der Netzwerke in eine zuweilen konflikthafte Spannung gerät (Löw und Knoblauch 2021). Ausdruck dieser Spannungen und Konflikte ist, dass den entgrenzenden Popularisierungen zuweilen mit sehr scharfen Markierungen der Grenzen des religiösen Feldes begegnet wird, zu deren Errichtung selbst modernste Kommunikationsmedien verwendet werden. Sie richten sich gegen die Auflösung von Religion ins Populäre, wie sie etwa in Fundamentalismen, Wiedererfindungen von Traditionen und Gegenbewegungen zu erkennen sind. Diese religiösen Bewegungen richten sich nicht mehr gegen die Moderne, sondern gegen die späte Moderne und ihre Durchsetzung von Vielfalt, Diversität und Teilhabe. 
Diese Grenzarbeiten (Gieryn 1983) bestätigen nicht nur die Rolle der Popularisierung sozusagen ex negativo. Sie zeigen auch, dass die populäre Religion als Teil einer umfassenderen Dynamik gefasst werden muss, die mit dem Konzept der Refiguration angeschnitten wird. Bezeichnenderweise ist die Religion noch immer oder wieder das Feld, in dem sich die Refiguration vielleicht am deutlichsten zeigt, und die populäre Religion ist vielleicht der klarste Ausdruck für die Tendenz zur Popularisierung von zunehmend mehr gesellschaftlichen Formen des (politischen, wissenschaftlichen, medizinischen etc.) Wissens (Knoblauch 2018) und für die damit einhergehenden Spannungen und Konflikte. Aus diesem Grund ist eine Fortführung von empirischen Untersuchungen der populären Religion ebenso wichtig wie deren präzise analytische Bestimmung.

Funding Open Access funding enabled and organized by Projekt DEAL.

Open Access Dieser Artikel wird unter der Creative Commons Namensnennung 4.0 International Lizenz veröffentlicht, welche die Nutzung, Vervielfältigung, Bearbeitung, Verbreitung und Wiedergabe in jeglichem Medium und Format erlaubt, sofern Sie den/die ursprünglichen Autor(en) und die Quelle ordnungsgemäß nennen, einen Link zur Creative Commons Lizenz beifügen und angeben, ob Änderungen vorgenommen wurden.

Die in diesem Artikel enthaltenen Bilder und sonstiges Drittmaterial unterliegen ebenfalls der genannten Creative Commons Lizenz, sofern sich aus der Abbildungslegende nichts anderes ergibt. Sofern das betreffende Material nicht unter der genannten Creative Commons Lizenz steht und die betreffende Handlung nicht nach gesetzlichen Vorschriften erlaubt ist, ist für die oben aufgeführten Weiterverwendungen des Materials die Einwilligung des jeweiligen Rechteinhabers einzuholen.

Weitere Details zur Lizenz entnehmen Sie bitte der Lizenzinformation auf http://creativecommons.org/ licenses/by/4.0/deed.de.

\section{Literatur}

Auslander, Philip. 1999. Liveness. Performance in a Mediatized Culture. London: Routledge.

Berger, Peter L. 1965. Ein Marktmodell zur Analyse ökumenischer Prozesse. In Internationales Jahrbuch für Religionssoziologie 1, 235-249.

Berger, Peter L. 1999. The desecularisation of the world. In The desecularisa-tion of the world: resurgent religion and world politics, Hrsg. Ders, 1-18. Washington, D.C.: Eerdmans.

Bourdieu, Pierre. 1985. Die Auflösung des Religiösen. In Rede und Antwort, Hrsg. Pierre Bourdieu, 231-237. Frankfurt a. M.: Suhrkamp.

Bruce, Steve. 2002. God is dead. Secularization in the west. Oxford: Blackwell.

Brunn, Frank Martin, und Sonja Keller. 2020. Teilhabe und Zusammenhalt. Potentiale von Religion im öffentlichen Raum. Leipzig: Evangelische Verlagsanstalt.

Burke, Peter. 1988. Popular culture in early modern Europe. Hants: Wildwood House.

Casanova, José. 1994. Public religions in the modern world. Chicago: University of Chicago Press.

Castells, Manuel. 1996. The rise of the network society. Malden: Blackwell.

Castells, Manuel. 2009. Communication power. Oxford: Oxford University Press.

Ebertz, Michael, und Franz Schultheis. 1986. Einleitung: populare Religiosität. In Volksfrömmigkeit in Europa. Beiträge zur Soziologie popularer Religiosität aus 14 Ländern, Hrsg. Michael Ebertz, Franz Schultheis, 16-28. Stuttgart: Kaiser.

Fiske, John. 1989. Understanding popular culture. London, New York: Routledge.

Forschungskonsortium WJT. 2007. Megaparty Glaubensfest. Weltjugendtag: Erlebnis - Medien - Organisation. Wiesbaden: VS.

Gebhardt, Winfried, Martin Engelbrecht, und Christoph Bochinger. 2005. Die Selbstermächtigung des religiösen Subjekts. Zeitschrift für Religionswissenschaft 2:133-152. 
Gerhards, Jürgen, und Friedhelm Neidhart. 1990. Strukturen und Funktionen moderner Öffentlichkeit. WZB Berlin: FS III., 90-101.

Gieryn, Thomas F. 1983. Boundary work and the demarcation of science from non-science: strains and interests in professional ideologies of scientists. American Sociological Review 48:781-795.

Greeley, Andrew. 1993. Religion in der Popkultur. Musik, Film und Roman. Graz: Styria.

Habermas, Jürgen. 2001. Glaube und Wissen. Frankfurt am Main: Suhrkamp.

Haken, Meike. 2017. Religiöse Kommunikation in der Konstruktion religiöser Großveranstaltungen. In Der kommunikative Konstruktivismus bei der Arbeit, Hrsg. Jo Reichertz, Rene Tuma, 160-187. Weinheim/Basel: Beltz Juventa.

Heelas, Paul, und Linda Woodhead. 2005. The spiritual revolution. Why religion is giving way to spirituality. Oxford: Blackwell.

Hepp, Andreas, und Veronika Krönert. 2009. Medien - Event-Religion. Die Mediatisierung des Religiösen. Wiesbaden: VS.

Isambert, Francois-André. 1982. Le sens du sacré. La religion populaire. Paris: Minuit.

Karstein, Uta. 2019. Wider die ,entadelte Kunst“ der Industrie. Zum Verhältnis von Kunst, Handwerk und Industrie im kirchlichen Milieu des 19. Jahrhunderts. In Räume zwischen Kunst und Religion. Sprechende Formen und religionshybride Praxis, Hrsg. Antje Mickan, Thomas Klie, und Peter A. Berger, 45-67. Bielefeld: transcript.

Kausch, Michael. 1988. Kulturindustrie und Populärkultur. Kritische Theorie der Massenmedien. Frankfurt am Main: Fischer.

Klinkhammer, Gritt, und Eva Tolksdorf (Hrsg.). 2015. Somatisierung des Religiösen. Empirische Studien zum rezenten religiösen Heilungs- und Therapiemarkt. Bremen: Universität Bremen.

Knoblauch, Hubert. 1989. Das unsichtbare neue Zeitalter. „New Age“, privatisierte Religion und kultische Milieus. Kölner Zeitschrift für Soziologie und Sozialpsychologie 41(3):504-525.

Knoblauch, Hubert. 1997. Die Sichtbarkeit der unsichtbaren Religion. Zeitschrift für Religionswissenschaft 5:179-202.

Knoblauch, Hubert. 1999. Berichte aus dem Jenseits. Wirklichkeit und Mythos der Nahtoderfahrung. Freiburg: Herder.

Knoblauch, Hubert. 2000. Populäre Religion. Markt, Medien und die Popularisierung der Religion. Zeitschrift für Religionswissenschaft 8:143-161.

Knoblauch, Hubert. 2002. Ganzheitliche Bewegungen, Transzendenzerfahrung und die Entdifferenzierung von Kultur und Religion in Europa. Berliner Journal für Soziologie 3:295-307.

Knoblauch, Hubert. 2009. Populäre Religion. Auf dem Weg in eine spirituelle Gesellschaft. Frankfurt a. M., New York: Campus.

Knoblauch, Hubert. 2010. Vom New Age zur populären Spiritualität. In Fluide Religion. Neue religiöse Bewegungen im Wandel. Theoretische und empirische Systematisierungen, Hrsg. Dorothea Lüddeckens, Rafael Walthert, 149-174. Bielefeld: transcript.

Knoblauch, Hubert. 2014. Benedict in Berlin. The mediatization of religion. In Mediatized worlds: culture and society in a media Age, Hrsg. Andreas Hepp, Friedrich Krotz, 143-158. London: Palgrave.

Knoblauch, Hubert. 2017. Die kommunikative Konstruktion der Wirklichkeit. Wiesbaden: VS.

Knoblauch, Hubert. 2018. Jenseits von Orthodoxie und Heterodoxie: das populäre Wissen. In Heterodoxie. Konzepte, Traditionen, Figuren der Abweichung, Hrsg. Michael Schetsche, Ina-Schmied-Knittel, 140-160. Köln: van Halem.

Knoblauch, Hubert. 2019. Bekenntnis, Konversion und kommunikative Konstruktion. In Bekenntnisse. Formen und Formeln, Hrsg. Christiane Bischoff, Carsten Juwig, und Lena Sommer, 17-37. Berlin: Reimer.

Knoblauch, Hubert. 2020. Die Refiguration der Religion. In Die Refiguration der Religion, Hrsg. Hubert Knoblauch. Weinheim/Basel: Beltz Juventa.

Knoblauch, Hubert, und Mathias Blanc. 2018. Bilder von Benedikt. Die Mediatisierung der Papstmesse und das religiöse Gefühl. In Das Bild als soziologisches Problem, Hrsg. Michael Müller, Hans-Georg Soeffner, 57-68. Weinheim, Basel: Beltz Juvenata.

Krotz, Friedrich, und Andreas Hepp (Hrsg.). 2012. Mediatisierte Welten. Wiesbaden: VS.

Löw, Martina, und Hubert Knoblauch. 2021. Digitale Mediatisierung und die Re-Figuration. In Räume digitaler Kommunikation, Hrsg. Thomas Döbler, Christian Petzold, und Christian Katzenbach. Köln: Van Halem.

Luckmann, Thomas. 1967. The invisible religion. New York: The Free Press.

Luckmann, Thomas. 1988. Die „massenkulturelle“ Sozialform der Religion. In Kultur und Alltag Sonderband 6 der „Sozialen Welt“.., Hrsg. H.G. Soeffner, 37-48. Göttingen: Schwartz \& Co.

Luckmann, Thomas. 1991. Die unsichtbare Religion. Frankfurt a. M.: Suhrkamp. 
Merle, Kristin. 2019. Religion in der Öffentlichkeit. Digitalisierung als Herausforderung für kirchliche Kommunikationskulturen. Berlin, New York: De Gruyter.

Neumaier, Anna. 2016. religion@home? Religionsbezogene Online-Plattformen und ihre Nutzung. Eine Untersuchung zu neuen Formen gegenwärtiger Religiosität. Würzburg: Ergon.

Reckwitz, Andreas. 2017. Die Gesellschaft der Singularitäten. Zum Strukturwandel der Moderne. Berlin: Suhrkamp.

Usinier, Jean-Claude, und Jörg Stolz (Hrsg.). 2014. Religions as brands. New perspectives on the marketization of religion and spirituality. Farnham: Ashgate. 